\title{
Orbital Correlations in Monolayer Manganites - From Spin $t-J$ Model to Orbital $t-J$ Model
}

\author{
M. DAGHOFER ${ }^{a}$ AND A.M. Oleśá ${ }^{a, b}$ \\ ${ }^{a}$ Max-Planck-Institut für Festkörperforschung \\ Heisenbergstr. 1, 70569 Stuttgart, Germany \\ ${ }^{b}$ Marian Smoluchowski Institute of Physics, Jagiellonian University \\ Reymonta 4, 30-059 Kraków, Poland
}

Dedicated to Professor Józef Spatek on the occasion of his 60th birthday

On the example of monolayer manganites we show that the theoretical ideas developed long ago along the derivation of the spin $t-J$ model from the Hubbard model are nowadays very helpful in strongly correlated oxides with partly filled degenerate orbitals. We analyze a realistic orbital $t-J$ model for $e_{\mathrm{g}}$ electrons in $\mathrm{La}_{1-x} \mathrm{Sr}_{1+x} \mathrm{MnO}_{4}$ monolayer manganites, and discuss the evolution of spin and orbital correlations under increasing doping by performing exact diagonalization of finite clusters, with electronic kinetic energy determined self-consistently at a finite temperature by classical Monte Carlo for $t_{2 \mathrm{~g}}$ spins. Several experimental results are reproduced.

PACS numbers: 75.10.Jm, 75.30.Et, 75.47.Lx

\section{Spin $t-J$ model}

A new perspective to treat strongly correlated electrons was opened by the derivation of the spin $t-J$ model three decades ago [1]. We argue that this derivation opened new routes to understand the complex magnetic and superconducting instabilities in the Hubbard model, and proved to be a very useful paradigm in strongly correlated electron systems. At that time it was already realized that the Hubbard model,

$$
\mathcal{H}_{0}=-t \sum_{i j \sigma} a_{i \sigma}^{\dagger} a_{j \sigma}+U \sum_{i} n_{i \uparrow} n_{i \downarrow}
$$

where $a_{i \sigma}^{\dagger}$ is an electron creation operator in spin state $\sigma=\uparrow, \downarrow$ at site $i$ and $U$ is local Coulomb interaction, is perfectly designed to describe electron localization 
in a Mott insulator, when the average filling is $n=1$ electron per site, and the Coulomb repulsion is large, i.e., $(t / U) \ll 1$. In this case the low-energy physics is determined by the effective superexchange interaction between localized electron states, which is antiferromagnetic (AF) due to the Pauli principle. In this limit the energy is determined by the nearest neighbor spin correlations and by higher order correction terms in powers of $(t / U)$ [2].

However, when a Mott insulator is doped by holes and electron density is reduced to $n=1-x$, the kinetic energy competes with the AF superexchange and may lead to novel physical phenomena [3]. Usually one considers then the effective spin $t-J$ model

$$
\mathcal{H}_{t J}=-t \sum_{i j \sigma} \tilde{a}_{i \sigma}^{\dagger} \tilde{a}_{j \sigma}+J \sum_{\langle i j\rangle} \boldsymbol{S}_{i} \cdot \boldsymbol{S}_{j}
$$

derived first in Ref. [1], where $\boldsymbol{S}_{i}$ are spin operators at each site occupied by a single electron, and $J=4 t^{2} / U$ is the superexchange constant. In contrast to Eq. (1), the kinetic energy term $\propto t$ contains "constrained" fermion creation operators, $\tilde{a}_{i \sigma}^{\dagger}=a_{i \sigma}^{\dagger}\left(1-n_{i \bar{\sigma}}\right)$ with $\bar{\sigma}=-\sigma$, and describes processes restricted to the subspace without doubly occupied sites. The lowest kinetic energy is obtained when spins are aligned in a ferromagnetic (FM) configuration. This competition between the kinetic and magnetic energy is the driving force responsible for the evolution of the magnetic phase diagram under increasing hole doping [4].

It was also recognized already in 1977 [1] that the faithful low-energy representation of the Hubbard model is not the $t-J$ model itself, but rather the $t-J$ model extended by the three-site term which contributes to the hole dynamics away from half-filling

$$
\mathcal{H}_{\mathrm{eff}}=-t \sum_{i j \sigma} \tilde{a}_{i \sigma}^{\dagger} \tilde{a}_{j \sigma}+J \sum_{\langle i j\rangle} \boldsymbol{S}_{i} \cdot \boldsymbol{S}_{j}-\frac{1}{4} J \sum_{i k j, \sigma \sigma^{\prime}} \tilde{a}_{i \sigma}^{\dagger} a_{k \sigma} n_{k \bar{\sigma}} n_{k \bar{\sigma}^{\prime}} a_{k \sigma^{\prime}}^{\dagger} \tilde{a}_{j \sigma^{\prime}}
$$

In fact, there is no reason to neglect this last term in Eq. (3) as it is of the same order as the effective exchange interaction $J[1]$, and plays an important role except when doping $x \rightarrow 0$. Indeed, the three-site term captures an important part of the dynamics near a Mott insulating state and is essential to give the correct increase in the Drude weight with doping $x[5]$, while a constant Drude weight follows instead from the $t-J$ model. The three-site term is also necessary to reproduce the doping dependence of the spectral weight for the lower and upper Hubbard bands in the optical spectra, while the $t-J$ model misses most of the spectral weight transfer between the Hubbard subbands which occurs due to kinetic processes [6]. For the same reason, only the complete form of the effective Hamiltonian Eq. (3) is appropriate to analyze yet another type of order in the regime of large $U$ - the superconducting phase. When the local pair operators, $b_{i j}=\left(\tilde{a}_{i \uparrow}^{\dagger} \tilde{a}_{i \downarrow}^{\dagger}-\tilde{a}_{i \downarrow}^{\dagger} \tilde{a}_{i \uparrow}^{\dagger}\right) / \sqrt{2}$, are introduced [7] for bond $\langle i j\rangle$, the effective Hamiltonian (3) describes correctly the dynamics of local pairs in a strongly correlated system. The three-site term gives an important contribution which favors the superconducting states over the 
magnetic ones, and could explain the observed increase in the critical temperature in the high $T_{\mathrm{c}}$ superconductors [7]. In contrast, when a propagation of a single hole in an AF background is analyzed, the $t-J$ model (2) is sufficient and gives new features in the electronic structure, such as quasiparticle band and incoherent part of the spectral density, which result from the coupling of a moving hole to spin excitations [8], while the three-site term leads only to quantitative corrections [9] which become important only at large $J \sim t[10]$. Therefore, the $t-J$ model is also extensively used for interpreting the data of photoemission experiments for high $T_{\mathrm{c}}$ superconductors at low hole doping [11]. In recent years the $t-J$ model continues to play an important role in the theoretical studies of high $T_{\mathrm{c}}$ superconductors, and serves to identify novel complex types of order [12].

Inter alia, the idea standing behind the $t-J$ model is extremely helpful to describe the doped correlated insulators with orbital degrees of freedom. One decade after the discovery of high $T_{\mathrm{c}}$ superconductivity the interest in strongly correlated systems has moved to a large extent to the systems with partly filled degenerate orbitals, where electron localization and magnetic interactions are rich and lead to complex phase diagrams [13]. In the last decade of the last century it was also realized that carriers in doped Mott-Hubbard insulators bind to $d-d$ excitations which gives rise to rather general and complex models of $t-J$ variety [14]. Both the derivation and theoretical treatment of such modes are a challenge in the modern theory of strongly correlated electrons. For instance, internal degrees of freedom of a moving carrier can change completely the behavior known from the spin $t-J$ model, where a singlet hole is responsible for coherent quasiparticles. In fact, a moving carrier with either orbital flavor or large spin (or both) leaves behind a path with its own history. Perhaps the simplest example of this behavior is provided by a triplet hole moving in an AF background [15], with its completely incoherent motion and suppressed the tendency towards FM polarons known from the singlet case [4].

Yet, there are also cases where the quasiparticle states are obtained by a coupling of the moving singlet hole to orbital excitations. The orbital $t-J$ model [16] was introduced in a close analogy to Eq. (2), and describes the motion of a hole in a FM plane of $\mathrm{LaMnO}_{3}$. The orbital superexchange $J$ follows again from virtual charge excitations in the regime of $U \gg t$, which applies to the realistic parameters of $\mathrm{LaMnO}_{3}$ [17]. As in the spin $t-J$ model [5], the three-site term plays here an important role near the half-filling (for weakly doped manganites), and is crucial to reproduce the correct behavior of the Drude weight at increasing doping $x$ [18]. Nevertheless, we neglect the three-site terms as they play almost no role at high doping $x \approx 0.5$, which is of interest in the present study.

For a FM plane it is allowed to decouple spin and orbital operators following the mean field procedure, although in general this would lead to incorrect results. In low-spin states the spin-orbital entanglement [19] generates novel ground states which do not follow from the classical expectations, such as the Goodenough- 
Kanamori rules [20]. The orbital order (OO) in an undoped insulator is already a challenge as the orbitals are directional, the orbital (superexchange) interactions depend on the bond direction [14], and their symmetry is thus cubic, i.e., much lower than the SU(2) symmetry in the spin case. As a result, the coherent quasiparticle states for a single hole moving in a Mott insulator with alternating orbital (AO) order have somewhat lower weight than in the spin case [16], and depend also on the type of the occupied orbitals.

Only during the last decade it has been recognized that the Jahn-Teller (JT) interactions and the superexchange, which occurs due to $e_{\mathrm{g}}$ electron excitations in the regime of large on-site Coulomb interaction $U$, support each other [17] and both are necessary to explain the magnetic and optical properties of undoped $\mathrm{LaMnO}_{3}$ [21]. In doped manganites, like in $\mathrm{La}_{1-x} \mathrm{Sr}_{x} \mathrm{MnO}_{3}$, the average density of $e_{\mathrm{g}}$ electrons $n=1-x$ is tuned by doping, and the paradigm of the orbital $t-J$ model may help to understand the actual orbital correlations in various doping regimes. In the present paper we follow this idea and analyze the orbital correlations in monolayer $\mathrm{La}_{1-x} \mathrm{Sr}_{1+x} \mathrm{MnO}_{4}$ manganites using the orbital $t-J$ model. The realistic model is richer than that of Ref. [16], and contains also the JT term [22], as well as the crystal field term which follows from the two-dimensional (2D) geometry and lifts the degeneracy of $e_{\mathrm{g}}$ orbitals. The orbital $t-J$ model constructed in this way includes all the terms which control the magnetic correlations in doped manganites [23].

The monolayer manganites are the subject of intense recent experimental research. The undoped monolayer $\mathrm{LaSrMnO}_{4}$ compound has the same magnetic structure as $\mathrm{K}_{2} \mathrm{NiF}_{4}$, with a 2D $G$-type AF $(G$-AF) order with AF moments in both directions [24]. At $x=0.5$ the AF order is CE-type (FM zig-zag chains with $\mathrm{AF}$ order in between), as deduced from the $\mathrm{X}$-ray and neutron scattering data for the structural and magnetic correlations [25], as well as from the neutron measurements of magnetic excitations [26]. One expects that the orbital correlations which follow from strong correlations and the JT interactions play an important role in stabilizing this phase [27]. Furthermore, unlike in $\mathrm{La}_{1-x} \mathrm{Sr}_{x} \mathrm{MnO}_{3}$, no FM metallic phase was found in doped monolayer $\mathrm{La}_{1-x} \mathrm{Sr}_{1+x} \mathrm{MnO}_{4}$ compounds, but instead short-range magnetic correlations [24, 25] indicate that magnetic interactions are frustrated. This behavior is puzzling and will be addressed in the present study.

\section{Orbital $t-J$ model}

We consider the effective orbital $t-J$ model,

$$
\mathcal{H}=H_{t}+H_{J}+H_{z}+H_{\mathrm{JT}}+H_{V}+H_{J^{\prime}},
$$

introduced here by generalizing the one-dimensional (1D) model of Ref. [22] to doped monolayer manganites. The first three terms are the same as the ones in the orbital $t-J$ model [16], while the other three serve to describe the realistic situation of $e_{\mathrm{g}}$ electrons in $\mathrm{La}_{1-x} \mathrm{Sr}_{1+x} \mathrm{MnO}_{4}$ manganites. The hopping term $H_{t}$ for monolayer manganites, 


$$
H_{t}=-\frac{1}{4} t \sum_{\langle i j\rangle \| a b} u_{i j}\left[3 \tilde{c}_{i x}^{\dagger} \tilde{c}_{j x}+\tilde{c}_{i z}^{\dagger} \tilde{c}_{j z} \mp \sqrt{3}\left(\tilde{c}_{i x}^{\dagger} \tilde{c}_{j z}+\tilde{c}_{i z}^{\dagger} \tilde{c}_{j x}\right)+\text { h.c. }\right]
$$

acts in the restricted space without double occupancies, so the operator $\tilde{c}_{i \alpha}^{\dagger}$ creates an electron in $|\alpha\rangle=|x\rangle,|z\rangle$ state at site $i$ only when this site is initially empty, i.e., $\tilde{c}_{i \alpha}^{\dagger}=c_{i \alpha}^{\dagger}\left(1-n_{i \bar{\alpha}}\right)$ with $\bar{\alpha}$ standing for the orbital favor opposite to $\alpha$, similar to spin in Eq. (2). We use here the conventional $e_{\mathrm{g}}$ orbital basis

$$
|x\rangle \equiv \frac{1}{\sqrt{2}}\left(x^{2}-y^{2}\right), \quad|z\rangle \equiv \frac{1}{\sqrt{6}}\left(3 z^{2}-r^{2}\right) .
$$

Here $t$ stands for an effective $(d d \sigma)$ element that originates from two $d-p$ transitions involving the intermediate oxygen ion. Following the double exchange mechanism [22], the hopping in Eq. (5) is modulated by the quantity $u_{i j}$ which depends on the spin state. Here the core spins $\boldsymbol{S}_{i}$ are treated as classical vectors of length $S$, so their state is represented by two polar angles $\left\{\vartheta_{i}, \phi_{i}\right\}$ per site, and the scalar product on the bond $\langle i j\rangle$ is given by

$$
\left\langle\boldsymbol{S}_{i} \cdot \boldsymbol{S}_{j}\right\rangle=S^{2}\left(2\left|u_{i j}\right|^{2}-1\right)
$$

where the actual dependence on the spins configuration gives

$$
\begin{aligned}
u_{i j} & =\cos \left(\frac{1}{2} \vartheta_{i}\right) \cos \left(\frac{1}{2} \vartheta_{j}\right)+\exp \left(\mathrm{i}\left(\phi_{j}-\phi_{i}\right)\right) \sin \left(\frac{1}{2} \vartheta_{i}\right) \sin \left(\frac{1}{2} \vartheta_{j}\right) \\
& =\exp \left(\mathrm{i} \chi_{i j}\right) \cos \left(\frac{1}{2} \theta_{i j}\right) .
\end{aligned}
$$

Therefore, in the classical approximation the hopping (5) is parameterized by the angle $\theta_{i j}$ between the two involved spins at sites $i$ and $j$, and by the complex phase $\chi_{i j}$.

The superexchange in $\mathrm{La}_{1-x} \mathrm{Sr}_{1+x} \mathrm{MnO}_{4}$ is given by a superposition of several terms, which originate from charge excitations by either $e_{\mathrm{g}}$ or $t_{2 \mathrm{~g}}$ electrons. The superexchange terms which follow from charge excitations by $e_{\mathrm{g}}$ electrons are included in $H_{J}$. These terms favor either FM or AF spin order on a considered bond $\langle i j\rangle$, depending on the pair of occupied $e_{\mathrm{g}}$ orbitals at both sites. The form of $H_{J}$ depends on the actual used orbital basis, and it is more convenient to use the directional orbitals and the corresponding pseudospin operators,

$$
T_{i}^{\zeta}=-\frac{1}{2}\left(T_{i}^{z} \mp \sqrt{3} T_{i}^{x}\right)
$$

depend on the bond direction with the sign - $(+)$ in Eq. (9) corresponding to $a$ (b) axis. The operators are defined by orbital $T=1 / 2$ pseudospin operators,

$$
T_{i}^{z}=\frac{1}{2} \sigma_{i}^{z}=\frac{1}{2}\left(\tilde{n}_{i x}-\tilde{n}_{i z}\right), \quad T_{i}^{x}=\frac{1}{2} \sigma_{i}^{x}=\frac{1}{2}\left(\tilde{c}_{i x}^{\dagger} \tilde{c}_{i z}+\tilde{c}_{i z}^{\dagger} \tilde{c}_{i x}\right)
$$

with two eigenstates of $T_{i}^{z}$, see Eq. (6).

The form of $H_{J}$ used below was derived in Ref. [22] from the complete spinorbital model [17]. It focuses on the orbital dynamics in the presence of spin fluctuations which tune orbital superexchange interactions. The spin operators on the bonds $\left(\mathrm{Mn}^{3+}-\mathrm{Mn}^{3+}\right.$ or $\left.\mathrm{Mn}^{3+}-\mathrm{Mn}^{4+}\right)$ are replaced by their expectation values which involve classical $u_{i j}$ parameters given in Eq. (8). In the present case of a monolayer one finds the $e_{\mathrm{g}}$ superexchange term [22], 


$$
\begin{aligned}
H_{J} & =J \sum_{\langle i j\rangle \| a b}\left\{\frac{1}{5}\left(2\left|u_{i j}\right|^{2}+3\right)\left(2 T_{i}^{\zeta} T_{j}^{\zeta}-\frac{1}{2} \tilde{n}_{i} \tilde{n}_{j}\right)-\frac{9}{10}\left(1-\left|u_{i j}\right|^{2}\right) \tilde{n}_{i \zeta} \tilde{n}_{j \zeta}\right. \\
& \left.-\left(1-\left|u_{i j}\right|^{2}\right)\left[\tilde{n}_{i \zeta}\left(1-\tilde{n}_{j}\right)+\left(1-\tilde{n}_{i}\right) \tilde{n}_{j \zeta}\right]\right\}
\end{aligned}
$$

where electron number operator $\tilde{n}_{i \zeta}$ refers in each case to the directional $\left(3 z^{2}-r^{2}\right)$-like orbital $|\zeta\rangle$ along a given bond $\langle i j\rangle$, e.g. $3 x^{2}-r^{2}$ orbital along $a$ axis. The last term in Eq. (11) stands for the $\mathrm{Mn}^{3+}-\mathrm{Mn}^{4+}$ superexchange and favors configurations of an occupied directional orbital next to a hole on the bond, where $\tilde{n}_{i}$ is the electron density operator in the restricted space. The superexchange constant $J=t^{2} / \bar{U}$ in Eq. (11) is determined by the lowest excitation energy $\bar{U}$ to the high-spin $S=5 / 2$ state of $d^{5}$ configuration at the involved $\mathrm{Mn}^{2+}$ ion [17].

The orbital $t-J$ model [16] contains also a uniform crystal field splitting $E_{z}>0$ of $e_{\mathrm{g}}$ orbitals which removes the orbital degeneracy in monolayer manganites, and is expected from the structural data [24]. Therefore, we use

$$
H_{z}=\frac{1}{2} E_{z} \sum_{i}\left(\tilde{n}_{i x}-\tilde{n}_{i z}\right),
$$

where $\tilde{n}_{i x}$ and $\tilde{n}_{i z}$ stand for the electron density operators in the two $e_{\mathrm{g}}$ orbitals. Again, as in the spin $t-J$ model, these density operators act in the restricted Hilbert space, with double occupancies projected out. If $E_{z}>0$, the $|z\rangle$ orbitals are favored as expected for the undoped $\mathrm{LaSrMnO}_{4}$ [24], and at $x=0.5$ doping in $\mathrm{La}_{0.5} \mathrm{Sr}_{1.5} \mathrm{MnO}_{4}[28]$.

The JT term $H_{\mathrm{JT}}$ includes two contributions

$$
H_{\mathrm{JT}}=\kappa \sum_{\langle i j\rangle \| a b} T_{i}^{\zeta} T_{j}^{\zeta}+\frac{1}{8} \kappa \sum_{\langle\langle i j\rangle\rangle \| a b} T_{i}^{\zeta}\left(1-\tilde{n}_{k}\right) T_{j}^{\zeta} .
$$

The first term stands for the orbital interaction between two $\mathrm{Mn}^{3+}$ ions on neighboring sites - it plays an important role in the undoped $\mathrm{LaSrMnO}_{4}$ and at low hole doping. The second one was derived from oxygen distortions by Bała et al. [29] and describes a second neighbor interaction along the bonds $\langle\langle i j\rangle\rangle$ parallel to either $a$ or $b$ axis; the coupling constant fixed here at $\frac{1}{8} \kappa$ was estimated from the distance dependence of the JT coupling. In addition, we also include the intersite Coulomb interaction between $e_{\mathrm{g}}$ electrons,

$$
H_{V}=V \sum_{\langle i j\rangle \| a b} \tilde{n}_{i} \tilde{n}_{j}
$$

- it plays a role in the doped regime, separating $e_{\mathrm{g}}$ electrons from each other.

Finally, the remaining part of the superexchange results from charge excitations by $t_{2 \mathrm{~g}}$ electrons between two $\mathrm{Mn}$ ions. Independently of their valence, the $t_{2 \mathrm{~g}}$ orbitals are filled by three electrons which form a core spin $S=3 / 2$ due to Hund's exchange $J_{\mathrm{H}}$. Therefore, this part of the superexchange is AF due to the Pauli principle, in analogy to the AF superexchange in the Hubbard model with nondegenerate orbitals. It can be verified by using the realistic parameters which 
describe the multiplet spectra of $\mathrm{Mn}$ ions that the different contributions to the AF superexchange are of similar value [30], so we describe the $t_{2 \mathrm{~g}}$ superexchange by the Heisenberg Hamiltonian $\left(J^{\prime}>0\right)$

$$
H_{J^{\prime}}=J^{\prime} \sum_{\langle i j\rangle}\left(\boldsymbol{S}_{i} \cdot \boldsymbol{S}_{j}-S^{2}\right) .
$$

For convenience, we consider here core spins $\boldsymbol{S}_{i}$ of unit length. Thereby the actual physical values of $S=3 / 2$ spins are compensated by a proper increase in $J^{\prime}$. The classical state of core spins $\left\{\boldsymbol{S}_{i}\right\}$ is given by two polar angles $\left\{\vartheta_{i}, \phi_{i}\right\}$ per site, which determine the energy following Eq. (7), and tune the kinetic energy (5) along each bond $\langle i j\rangle$.

The ground state of a monolayer obtained at a given doping $x$ is characterized by electron density distribution which we quantify by average electron densities $\left\{n_{x}, n_{z}\right\}$ in orbitals $\alpha=x, z$. The competing tendencies between FM and AF spin correlations at short distances, coexisting with different types of OO, have been investigated by intersite spin and orbital correlations at distance $\boldsymbol{r}$ :

$$
\mathcal{S}(\boldsymbol{r})=\frac{1}{N} \sum_{i}\left\langle\boldsymbol{S}_{i} \cdot \boldsymbol{S}_{i+\boldsymbol{r}}\right\rangle, \quad \mathcal{T}_{\theta}(\boldsymbol{r})=\frac{1}{N} \sum_{i}\left\langle T_{i}(\theta) T_{i+\boldsymbol{r}}(\theta)\right\rangle .
$$

The orbital operators are defined for a particular orbital basis at site $i$,

$$
T_{i}(\theta)=T_{i}^{z} \cos \theta+T_{i}^{x} \sin \theta
$$

and the pseudospin operators $\left\{T_{i}^{z}, T_{i}^{x}\right\}$ are given by Eqs. (10). For instance, $\theta=0$ corresponds to $T_{i}^{z} T_{i+m}^{z}, \theta=\pi / 2-$ to $T_{i}^{x} T_{i+m}^{x}$, and $\theta=2 \pi / 3-$ to $T_{i}^{\zeta} T_{i+\boldsymbol{r}}^{\zeta}$, with $\zeta$ standing for the directional orbital along $a$ axis. The orbital correlations expected in undoped manganites are of AO type on two sublattices, which suggests that the orbital correlations $\mathcal{T}_{\theta}(\boldsymbol{r})$ defined as in Eq. (17) are predominantly negative for nearest neighbors. These correlations were investigated along the (10) and (11) (and equivalent) directions in 2D clusters.

\section{Numerical results}

We employed two different numerical methods to investigate finite clusters described by the orbital $t-J$ model (4): (i) exact diagonalization at zero temperature $(T=0)$ for fixed core spin configurations (FM, $G$-AF, $C$-AF with FM order in one and $\mathrm{AF}$ in the other direction, and CE phase), and (ii) Markov chain Monte Carlo (MC) at a finite temperature $T>0$ for core spins [31], combined with exact diagonalization for the $e_{\mathrm{g}}$ orbital problem. The ground state at $T=0$ for representative electron fillings was determined by solving the orbital $t-J$ model for several possible types of spin order, using $\sqrt{8} \times \sqrt{8}$ and $4 \times 4$ clusters with periodic boundary conditions. We solved the orbital problem obtained for the selected core spin configuration using the Lanczos algorithm. We then determined the global ground state by comparing the energies obtained for different magnetic phases.

At finite temperatures, we investigated the effective orbital $t-J$ model (4) by making use of a combination of the Markov chain MC algorithm for the core 
spins [31] with Lanczos diagonalization of the orbital problem. For each classical core spin configuration occurring in the MC runs, we defined the actual values of classical variables $\left\{u_{i j}\right\}$, and next solved the orbital model. In each case we obtained the free energy for that core spin configuration from the few lowest eigenstates, which was next used to decide acceptance in the MC runs. More details about the calculation method were given in Ref. [32].

We begin with the phase diagram obtained for the undoped $\mathrm{LaSrMnO}_{4}$. In the absence of holes the hopping in Eq. (5) is blocked, and the Coulomb interaction $V$ plays no role as it simply adds a constant energy for each bond. Therefore, the ground state for the finite $J$ is determined by only three parameters: $E_{z} / J, \kappa / t$, and $J^{\prime} / J$. The first two parameters determine the orbital state, and the last one tunes the spin interactions. The magnetic order depends on all these parameters as the superexchange due to $e_{\mathrm{g}}$ electrons is intrinsically frustrated and contains both AF and FM terms [17], which become active for particular pairs of occupied orbitals on a given bond $\langle i j\rangle$, see Eq. (11).

Apart from the unphysical regime of negative $J^{\prime}<0$ which enforces the FM order independently of the orbital state, the AO order is necessary to stabilize it for $J^{\prime} \approx 0$ and $\left|E_{z}\right|<0.5 t$. This agrees with the Goodenough-Kanamori rules [20], and with the interplay between the magnetic and $e_{\mathrm{g}}$ orbital correlations studied before in the $1 \mathrm{D}$ model [22]. The FM phase competes with the $G$-AF order stable for the sufficiently large core spin superexchange $J^{\prime}$ (Fig. 1a). However, the transition line depends also on the two remaining parameters: $E_{z}$ and $\kappa$. The dependence on $E_{z}$ is stronger - for $E_{z}<-0.5 t$ the AF order is stable already at $J^{\prime}=0$, while for $E_{z}>0.5 t$ the monolayer would be FM, if the AF superexchange $J^{\prime}$ between core spins would not help to stabilize the AF order. This kind of behavior is a manifestation of the large difference of 9:1 between the $\mathrm{AF} e_{\mathrm{g}}$ superexchange
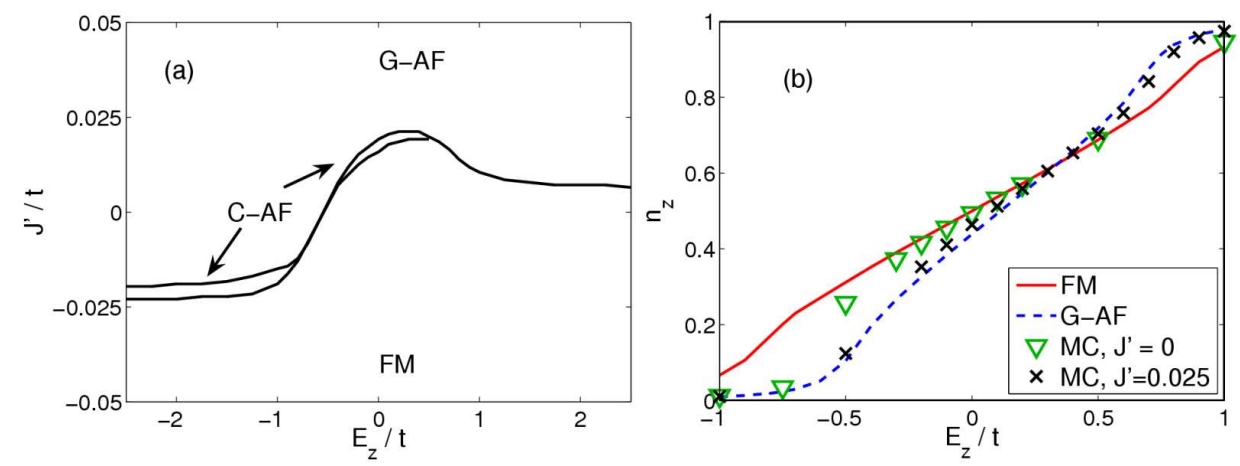

Fig. 1. Magnetic and orbital state for the undoped monolayer $(x=0)$ obtained with a $\sqrt{8} \times \sqrt{8}$ cluster: (a) phase diagram with the stability regions of FM, $G$-AF, and $C-\mathrm{AF}$ phases in the $\left(E_{z}, J^{\prime}\right)$ plane at $T=0$; (b) electron density $n_{z}$ in out-of-plane $|z\rangle$ orbitals for increasing value of the crystal field splitting $E_{z}$, as obtained for the FM and AF ground states (solid and dashed line), as well as the MC data for $\beta t=100$ and two values of $J^{\prime}$. Parameters: $J=0.125 t, \kappa=0.2 t$. 
interaction [33], depending on whether $e_{\mathrm{g}}$ electrons are in $|x\rangle$ or in $|z\rangle$ orbitals, respectively. Altogether, the transition line between the $\mathrm{FM}$ and $G$-AF phase is only weakly dependent on $E_{z}$ in the regime of large $\left|E_{z}\right|>t$; once all electrons are found in either $|x\rangle$ or $|z\rangle$ ferro orbital (FO) phase, a further increase in $\left|E_{z}\right|$ does not change the state. Remarkably, the range of $C$-AF phase is very narrow and vanishes for $E_{z}>0.3 t$, indicating that a large overlap between $|x\rangle$ orbitals plays a role in stabilizing this type of order, which could also be a finite size effect.

At the expected value of core spin superexchange $J^{\prime}=0.025 t$ in $\mathrm{LaSrMnO}_{4}$, the $G$-AF state is found independently of $E_{z}$ (Fig. 1b). However, the experimental data suggest that $|z\rangle$ orbitals are occupied in $\mathrm{LaSrMnO}_{4}$ [24], and we find that this situation is well described by $E_{z} \sim t$. When temperature increases to $\beta t=100$, the orbital state almost does not change and indeed the MC data for the density $n_{z}$ follow the line obtained at $T=0$ for $G$-AF phase, see Fig. 1b. A further increase in temperature promotes excitations to $|x\rangle$ orbitals [24], and this experimental finding is also reproduced by the present model [34]. It is interesting to remark that the $G$ - $\mathrm{AF}$ order is stabilized in this parameter regime by the core spin $\mathrm{AF}$ superexchange $J^{\prime}$, while the $e_{\mathrm{g}}$ part of the superexchange is $\mathrm{FM}$, as the excitations from occupied $|z\rangle$ to unoccupied $|x\rangle$ orbitals dominate, with the hopping element larger by $\sqrt{3}$ than the one between two $|z\rangle$ orbitals, which would give instead the AF coupling.

At $J^{\prime}=0$ the FM state is stable for $E_{z}>0$, but particularly when the electron densities in both $e_{\mathrm{g}}$ orbitals are similar for $E_{z} \approx 0$, and the $\mathrm{OO}$ may form with occupied orbitals

$$
\begin{aligned}
|\theta\rangle_{i \in \mathrm{A}} & =\cos \left(\frac{1}{2} \theta\right)|z\rangle_{i}+\sin \left(\frac{1}{2} \theta\right)|x\rangle_{i}, \\
|\theta\rangle_{j \in \mathrm{B}} & =\cos \left(\frac{1}{2} \theta\right)|z\rangle_{j}-\sin \left(\frac{1}{2} \theta\right)|x\rangle_{j},
\end{aligned}
$$

on two sublattices A and $\mathrm{B}$. In this case the FM state is robust and rather large $J^{\prime} \approx 0.025 t$ is necessary to switch the magnetic correlations to the $G$-AF state (Fig. 1a). This is also shown by the MC data for the electron density in $|z\rangle$ orbitals $n_{z}$, which remains practically unchanged from the ground state value still at temperature $\beta t=100$ (Fig. 1b). The density $n_{z}$ increases slower with $E_{z}$ than in the AF state, as the AO state (18) can occur only when the electron density in $|z\rangle$ orbitals is similar to that in $|x\rangle$ orbitals. Let us note also that a rapid drop of $n_{z}$ with decreasing $E_{z}$ near $E_{z}=-0.5 t$ in the MC data for $J^{\prime}=0$ accompanies the phase transition to the $G$-AF phase, see the phase boundary in Fig. 1a.

We emphasize that, similar to $\mathrm{LaMnO}_{3}[17]$, the AO state with FM spin order is stabilized not only by the orbital superexchange terms in Eq. (11), but also by the orbital JT interactions (13). This results in the separation of the energy scales at finite $\kappa=0.2 t$ shown in Fig. 2 - the magnetic order is lost first in the range of temperature $\beta t \approx 50$, while the $\mathrm{AO}$ order is more robust and starts to weaken only above $\beta t \approx 10$. While at $\kappa=0$ the orbital correlations decrease somewhat faster with increasing temperature [34], they are still quite pronounced when the 

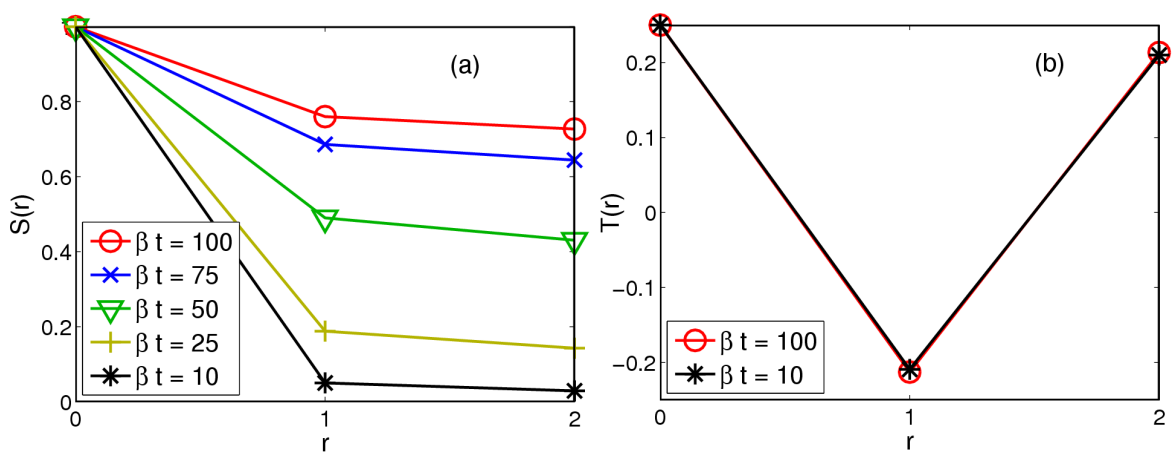

Fig. 2. Short range correlations for the undoped monolayer manganite $(x=0)$ as obtained in the FM state with a $\sqrt{8} \times \sqrt{8}$ cluster for increasing temperature: (a) spin, (b) orbital for $\theta=\pi / 2$. Parameters: $J=0.125 t, \kappa=0.2 t$, and $J^{\prime}=0$.

magnetic order is lost. This shows that FM correlations would be typically lost at a lower temperature $(T \approx 150 \mathrm{~K})$ than the orbital correlations (see Fig. $2 \mathrm{~b}$ ), to a large extent due to conflicting trends in magnetic interactions which compete with each other.

In order to understand the evolution of magnetic interactions with increasing hole doping, it is instructive to consider first the orbital occupation. The geometry of the monolayer compounds suggests positive crystal field splitting $E_{z}>0$ of $e_{\mathrm{g}}$ orbitals, so the $|z\rangle$ orbitals which stick out of the $a b$ plane are favored. We have found that for reasonable positive values of $E_{z}$ the majority of electrons is still in $|z\rangle$ orbitals at low doping, see Fig. 3. However, for $x>0.2$ both $e_{\mathrm{g}}$ orbitals are either nearly equally populated, or the $|x\rangle$ orbitals dominate, i.e., $n_{x}>n_{z}$. This behavior confirms the trend found before in the $2 \mathrm{D}$ model at orbital degene-

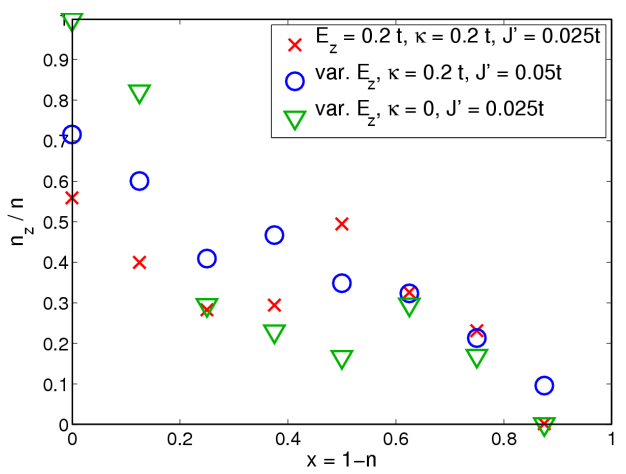

Fig. 3. Percentage of electrons found in $|z\rangle$ orbitals $n_{z} / n$ as a function of doping $x$ obtained by MC on a $\sqrt{8} \times \sqrt{8}$ cluster for three parameter sets: (i) $E_{z}=0.2 t, \kappa=0.2 t$, $J^{\prime}=0.025 t$, (ii) variable $E_{z}=\frac{1}{2}(1-x), \kappa=0.2 t, J^{\prime}=0.05 t$, and (iii) variable $E_{z}=\left(\frac{1}{2}-x\right), \kappa=0, J^{\prime}=0.025 t$ (the last set of data has also been shown in Ref. [32]). Parameters: $J=0.125 t, V=t$. 
racy [35]. Here we show that the FO order with $|x\rangle$ orbitals occupied cannot form as long as crystal field splitting is not lower than $E_{z} \approx 0$. This helps to suppress the kinetic energy in $a b$ plane, so the double exchange interaction is weak at finite doping. Actually, for this reason the FM state does not occur in the range of intermediate doping $0.2<x<0.4$ when $E_{z}>0$ and the core spin superexchange is in the expected range, $J^{\prime} \sim 0.05 t$. Altogether, we argue that the orbital liquid state which forms only in cubic geometry [36] plays an essential role in stabilizing the FM state.

We believe that the crystal field splitting gradually decreases with increasing $e_{\mathrm{g}}$ hole density, as the distortions of $\mathrm{MnO}_{6}$ octahedra are then expected to decrease and could vanish in the limit of $x=1$. Qualitatively this situation is modelled by variable $E_{z}=\frac{1}{2}(1-x) t$ in Fig. 3 . While about $70 \%$ of electrons fill then $|z\rangle$ orbitals at $x=0$, the filling of these orbitals at $x=0.5$ is still about $35 \%$ (for $E_{z}=\frac{1}{4} t$ ). In contrast, when $E_{z}=0$ (last set of data in Fig. 3), the filling of $|z\rangle$ orbitals drops below $20 \%$, being too low to form the observed OO state [28]. Below we analyze the influence of the electron density distribution and the microscopic parameters on the stability of the CE-AF phase at $x=0.5$.

The CE phase was observed in a monolayer $\mathrm{La}_{0.5} \mathrm{Sr}_{1.5} \mathrm{MnO}_{4}$ compound [25], corresponding to half doping $(x=0.5)$. This type of order is quite exotic and there were several attempts in the literature to identify the leading parameters which stabilize this complex type of coexisting charge, orbital, and magnetic order. We have performed extensive ground state $(T=0)$ calculations using $\sqrt{8} \times \sqrt{8}$ clusters with periodic boundary conditions, which are large enough to capture the essential interactions deciding about the nature of the ground state [29], as we also concluded recently [32].

In the absence of JT interactions (at $\kappa=0$ ), the $\mathrm{CE}$ phase arises as a compromise between competing $\mathrm{AF}$ and $\mathrm{FM}$ interactions, roughly speaking in a range of $0<J^{\prime}<0.1 t$ and $V<1.5 t$ [32]. In contrast to the undoped case, the magnetic interactions at half doping are in general anisotropic and the orbital state can be then tuned to support FM spin order along a certain direction by double exchange mechanism, while the superexchange is predominantly AF in the other direction. In this way the $C$-AF (studied before in the ladder geometry [37]) and CE-AF phase may arise and strongly compete with each other due to rather similar kinetic and interaction energies [32], as long as $\kappa=0$. The situation changes however when the JT interactions are considered, as finite $\kappa=0.2 t$ used for the data of Fig. 4a favors also alternation of two quasi-planar orbitals,

$$
|+\rangle_{i \in \mathrm{A}}=\frac{1}{\sqrt{2}}\left(|z\rangle_{i}+|x\rangle_{i}\right), \quad|-\rangle_{j \in \mathrm{B}}=\frac{1}{\sqrt{2}}\left(|z\rangle_{i}-|x\rangle_{i}\right)
$$

on the charge majority sites in the regime of $0<E_{z}<0.5 t$, where electron densities in $|x\rangle$ and $|z\rangle$ orbital are similar, rather than directional $3 x^{2}-r^{2} / 3 y^{2}-r^{2}$ orbitals. For these parameters the $C$-AF phase is destabilized. Thus, for a moderate value of core spin superexchange $J^{\prime}=0.025 t$, the CE phase competes only with the FM 

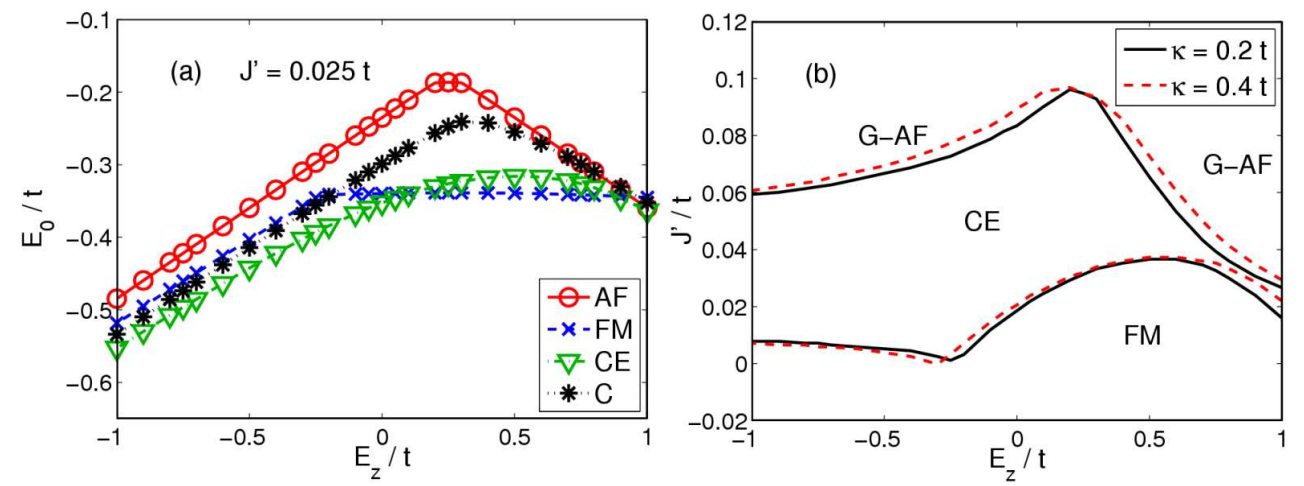

Fig. 4. Evolution of the magnetic ground state for $x=0.5$ doping as obtained with a $\sqrt{8} \times \sqrt{8}$ cluster for increasing crystal field splitting $E_{z}$ of $e_{\mathrm{g}}$ orbitals: (a) energies for various possible magnetic phases $(G-\mathrm{AF}, \mathrm{FM}, \mathrm{CE}-\mathrm{AF}, C-\mathrm{AF})$ for $\kappa=0.2 t$ and $J^{\prime}=0.025 t$; (b) phase diagram in $\left(E_{z}, J^{\prime}\right)$ plane for $\kappa=0.2 t$ (solid lines) and $\kappa=0.4 t$ (dashed lines). Parameters: $J=0.125 t, V=t$.

phase, and the latter one takes over for $E_{z}>0.15 t$, i.e., when the population of both $e_{\mathrm{g}}$ orbitals is almost equal and the double exchange energy dominates. This is also seen in the phase diagram of Fig. $4 \mathrm{~b}$ - increasing $E_{z}$ pushes the CE phase to higher values of $J^{\prime}$ in a range of $-0.25 t<E_{z}<0.5 t$. Also the transition between the $\mathrm{CE}$ and $G$-AF occurs at somewhat higher values of $J^{\prime}$ when $E_{z}$ increases in a range of $-t<E_{z}<0.25 t$. Quite remarkably, when $E_{z}$ increases beyond these limits, an opposite trend is found, as then only $|z\rangle$ orbitals are occupied and the OO which accompanies the CE phase (19) cannot develop. Therefore, the CE phase collapses, and may exist only in a very narrow window of $J^{\prime}$ values in between the FM and $G$-AF phase.
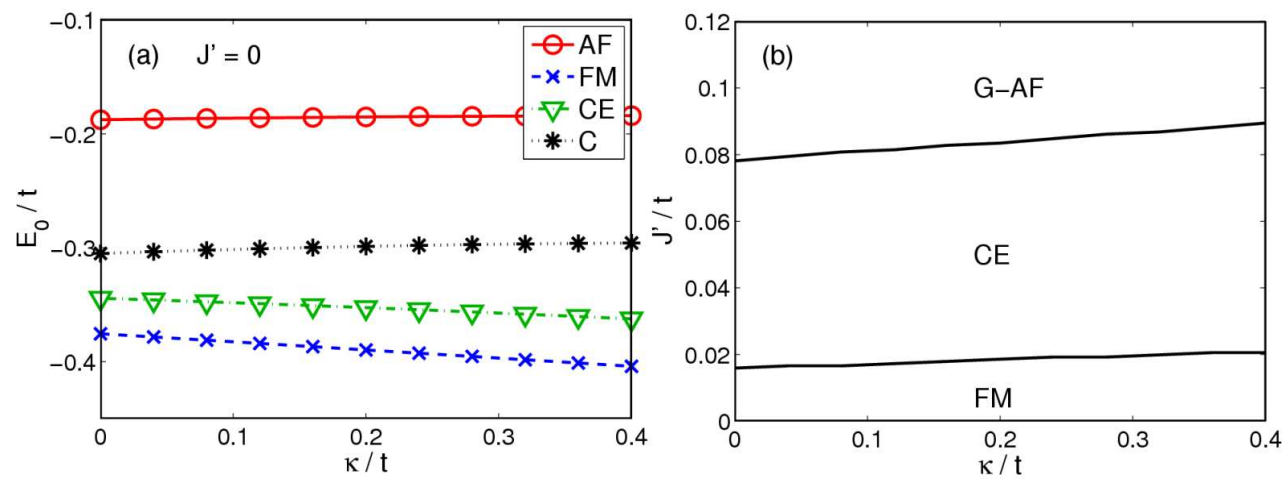

Fig. 5. Magnetic phases for increasing JT interaction $\kappa$ as obtained with a $\sqrt{8} \times \sqrt{8}$ cluster for $x=0.5$ : (a) ground state energies of various phases ( $G$-AF, FM, CE-AF, $C$-AF) for $J^{\prime}=0$; (b) phase diagram in $\left(\kappa, J^{\prime}\right)$ plane. Parameters: $J=0.125 t, E_{z}=0$, and $V=t$. 
Surprisingly, the dependence of all magnetic energies on the value of the JT interaction $\kappa$ is quite weak, see Fig. 5. This interaction stabilizes the OO both in the FM and in CE phase, so the energies of these phases decrease with increasing $\kappa$ (Fig. 5a). However, the actual border lines between these two phases and between the $\mathrm{CE}$ and $G$-AF phase, respectively, are determined by the value of $J^{\prime}$ (Fig. $5 \mathrm{~b}$ ), and depend also on the electron density distribution over $|x\rangle$ and $|z\rangle$ orbitals, as discussed above.

We also note that a finite value of $V \sim t$ is important and helps to stabilize the checkerboard charge order coexisting with the $\mathrm{OO}$ in the $\mathrm{CE}$ phase. As expected in this state, the $|+\rangle$ and $|-\rangle$ occupied orbitals (19) alternate on the sites with increased electron density when $V=t$ and $\kappa=0.2 t$ (Fig. 6). This OO is well developed, but still rather far from the idealized one, as $e_{\mathrm{g}}$ electrons are partly delocalized. We note that this partial delocalization is crucial for the double exchange which favors the FM order along the zigzag chain.

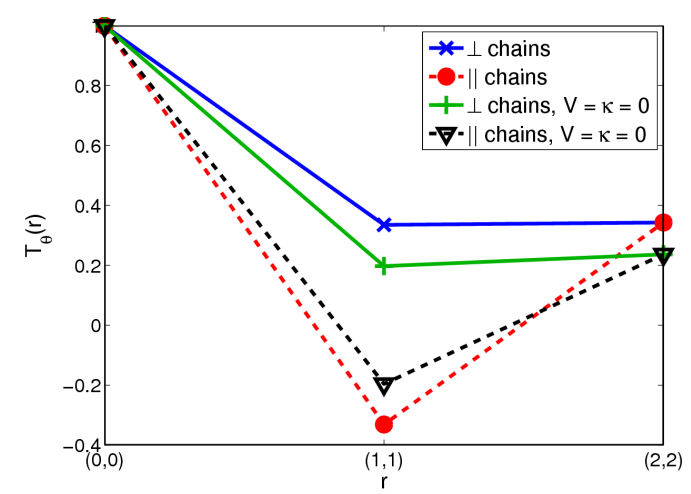

Fig. 6. Orbital correlations along the diagonal $(1,1)$ and $(1, \overline{1})$ directions in the $\mathrm{CE}$ phase $(x=0.5)$ for $J=0.125 t$, and for: $V=t, \kappa=0.2 t$ (solid lines), and $V=\kappa=0$ (dashed lines).

Although the competition between the AF superexchange and the FM double exchange persists also at higher doping $x>0.5$, the checkerboard charge ordered state is destabilized, and the optimal conditions for the CE phase are not fulfilled. Instead, one finds a robust tendency towards the $C$-AF phase, as we demonstrate by the data obtained with a $4 \times 4$ cluster for doping $x=0.75$ in Fig. 7a. As for $x=0.5$, the FM phase is stable at $J^{\prime}=0$. In this case the density of $e_{\mathrm{g}}$ electrons is so low that the AF superexchange between $\mathrm{Mn}^{3+}$ and $\mathrm{Mn}^{4+}$ ions is rather weak and the $C$-AF phase, observed experimentally in $\mathrm{Nd}_{1-x} \mathrm{Sr}_{1+x} \mathrm{MnO}_{4}$ [38], is obtained only when $J^{\prime}>0.02 t$, i.e., in the expected range obtained by analyzing the $t_{2 \mathrm{~g}}$ superexchange. The value of critical $J^{\prime}$ decreases somewhat with increasing $E_{z}$, as the double exchange weakens when $|z\rangle$ orbitals are occupied for positive values of $E_{z}$. This effect is quite strong for the second phase boundary, and one finds 

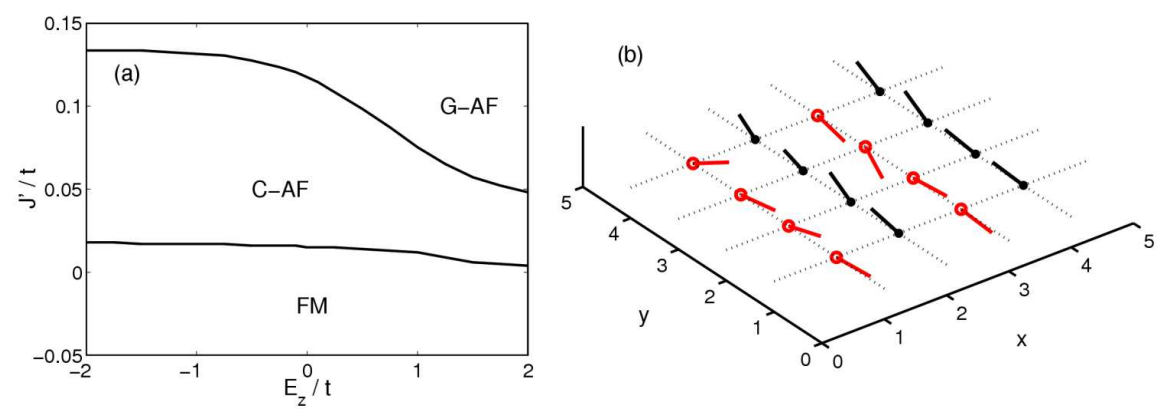

Fig. 7. Competition between AF and FM spin interaction at $x=0.75$ doping, as obtained with a $4 \times 4$ cluster: (a) phase diagram in $\left(E_{z}, J^{\prime}\right)$ plane with FM, $C$-AF, and $G$-AF phases; (b) MC snapshot of the $C$-AF phase for $E_{z}=t, J^{\prime}=0.05 t, \beta t=100$. Other parameters: $J=0.125 t, \kappa=0.2 t$, and $V=t$.

that the $C$-AF phase survives in a considerably broader range of $J^{\prime}$ when $E_{z}<0$. Actually, the critical value of $J^{\prime}$ exchange changes roughly by a factor of three between $E_{z}=-2 t$ and $E_{z}=+2 t$, which originates from the strength of the double exchange proportional to the respective hopping elements between two $|x\rangle$ or two $|z\rangle$ orbitals, respectively.

\section{Discussion and summary}

The present study clarifies that orbital degrees of freedom are of crucial importance for a complete understanding of magnetic correlations in monolayer manganites. We treated a realistic orbital $t-J$ model including Coulomb and JT interactions and investigated charge, spin, and orbital intersite correlations in monolayer manganites. The obtained results revealed a close relationship between orbital and magnetic order which follows the classical Goodenough-Kanamori rules at $x=0[20]$. The magnetic phases found in different doping regimes, where double exchange also contributes, are in accordance with experiments over the entire doping range $0 \leq x<1$.

For the undoped monolayer $\mathrm{LaSrMnO}_{4}$ manganite the model predicts $G$-AF order, when the crystal field splitting of $e_{\mathrm{g}}$ orbitals $E_{z} \sim t$ and the core spin superexchange $J^{\prime} \sim 0.03 t$ are in the expected range. Due to large crystal field splitting the role played here by the intersite orbital interactions $\propto \kappa$ is in this case negligible. We emphasize that the correct treatment of electron correlation effects within the orbital $t-J$ model [16] is crucial for the qualitatively correct description of the magnetic state in $\mathrm{LaSrMnO}_{4}$, and quite different results are obtained when strong on-site Coulomb repulsion $U$ is neglected [39].

Another success of the model is that it predicts the CE phase at half doping with physically realistic parameters for layered manganites, i.e., for small $t_{2 \mathrm{~g}}$ superexchange $J^{\prime} \sim 0.03 t$, as deduced [21] from the analysis of exchange constants in $\mathrm{LaMnO}_{3}$, rather than for unrealistically large $J^{\prime}>0.2 t$ [40]. The orbital inter- 
actions promoted by the JT effect play here a very important role in stabilizing this phase, as they support precisely the type of the AO order on the checkerboard lattice which promotes FM interactions along the zigzag chains by double exchange mechanism. The occupied orbitals are closer to the planar $z^{2}-x^{2} / y^{2}-z^{2}$ orbitals [28], rather than to the directional $3 x^{2}-r^{2} / 3 y^{2}-r^{2}$ orbitals, which could also be explained by a crystal field $E_{z}>0$ [32], as is frequently believed. Also at doping $x \approx 0.75$, the present orbital $t-J$ model predicts the $C$-AF phase in agreement with experiment [38].

Summarizing, the present study shows that the magnetic phase diagrams of monolayer manganites demonstrate a competition between different types of order which follows from coexisting FM and AF terms in the spin superexchange, and the FM double exchange at finite hole doping. At the magnetic transitions the orbital order changes simultaneously, as it supports particular types of magnetic order, in agreement with the Goodenough-Kanamori rules. We find it quite remarkable that yet another application of the ideas developed about thirty years ago along the derivation of the celebrated spin $t-J$ model [1] turned out to be so successful in monolayer manganites, and reproduced several generic features observed experimentally in this class of strongly correlated electron systems.

\section{Acknowledgments}

We thank Peter Horsch for insightful discussions. A.M. Oleś acknowledges support by the Polish Ministry of Science and Education under project No. N202 068 32/1481.

\section{References}

[1] K.A. Chao, J. Spałek, A. M. Oleś, J. Phys. C 10, L271 (1977).

[2] K.A. Chao, J. Spałek, A.M. Oleś, Phys. Rev. B 18, 3453 (1978).

[3] E. Dagotto, Rev. Mod. Phys. 66, 763 (1994); J. Spałek, Acta Phys. Pol. A 111, 409 (2007).

[4] J. Spałek, A.M. Oleś, K.A. Chao, Phys. Status Solidi B 108, 329 (1981).

[5] W. Stephan, P. Horsch, Int. J. Mod. Phys. B 6, 589 (1992).

[6] H. Eskes, A.M. Oleś, Phys. Rev. Lett. 73, 1279 (1994).

[7] J. Spałek, Phys. Rev. B 37, 533 (1988).

[8] G. Martínez, P. Horsch, Phys. Rev. B 44, 317 (1991).

[9] K.J. von Szczepanski, P. Horsch, W. Stephan, M. Ziegler, Phys. Rev. B 41, 2017 (1990).

[10] J. Bała, A.M. Oleś, J. Zaanen, Phys. Rev. B 52, 4597 (1995).

[11] A. Damascelli, Z. Hussain, Z.-X. Shen, Rev. Mod. Phys. 75, 473 (2003).

[12] M. Raczkowski, D. Poilblanc, R. Frésard, A.M. Oleś, Phys. Rev. B 75, 094505 (2007).

[13] A. Klejnberg, J. Spałek, Phys. Rev. B 57, 12041 (1998); 61, 15542 (2000). 
[14] J. Zaanen, A.M. Oleś, Phys. Rev. B 48, 7197 (1993).

[15] J. Zaanen, A.M. Oleś, P. Horsch, Phys. Rev. B 46, 5798 (1992).

[16] J. van den Brink, P. Horsch, A.M. Oleś, Phys. Rev. Lett. 85, 5174 (2000).

[17] L.F. Feiner, A.M. Oleś, Phys. Rev. B 59, 3295 (1999).

[18] P. Horsch, J. Jaklič, F. Mack, Phys. Rev. B 59, 6217 (1998).

[19] A.M. Oleś, P. Horsch, L.F. Feiner, G. Khaliullin, Phys. Rev. Lett. 96, 147205 (2006).

[20] J.B. Goodenough, Magnetism and the Chemical Bond, Interscience, New York 1963; J. Kanamori, J. Phys. Chem. Solids 10, 87 (1959).

[21] A.M. Oleś, G. Khaliullin, P. Horsch, L.F. Feiner, Phys. Rev. B 72, 214431 (2005).

[22] M. Daghofer, A.M. Oleś, W. von der Linden, Phys. Rev. B 70, 184430 (2004).

[23] A. Weisse, H. Fehske, New J. Phys. 6, 158 (2004).

[24] D. Senff, P. Reutler, M. Braden, O. Friedt, D. Bruns, A. Cousson, F. Bourée, M. Merz, B. Büchner, A. Revcolevschi, Phys. Rev. B 71, 024425 (2005).

[25] S. Larochelle, A. Mehta, L. Lu, P.K. Mang, O.P. Vajk, N. Kaneko, J.W. Lynn, L. Zhou, M. Greven, Phys. Rev. B 71, 024435 (2005).

[26] D. Senff, F. Krüger, S. Scheidl, M. Benomar, Y. Sidis, F. Demmel, M. Braden, Phys. Rev. Lett. 96, 257201 (2006).

[27] Y.S. Lee, S. Onoda, T. Arima, Y. Tokunaga, J.P. He, Y. Kaneko, N. Nagaosa, Y. Tokura, Phys. Rev. Lett. 97, 077203 (2006).

[28] S.B. Wilkins, N. Stojic, T.A.W. Beale, N. Binggeli, C.W.M. Castleton, P. Bencok, D. Prabhakaran, A.T. Boothroyd, P.D. Hatton, M. Altarelli, Phys. Rev. B 71, 245102 (2005).

[29] J. Bała, P. Horsch, F. Mack, Phys. Rev. B 69, 094415 (2004).

[30] A.M. Oleś, L.F. Feiner, Phys. Rev. B 65, 052414 (2002).

[31] E. Dagotto, S. Yunoki, A.L. Malvezzi, A. Moreo, J. Hu, S. Capponi, D. Poilblanc, N. Furukawa, Phys. Rev. B 58, 6414 (1998).

[32] M. Daghofer, A.M. Oleś, D. Neuber, W. von der Linden, Phys. Rev. B 72, 104451 (2006).

[33] A.M. Oleś, L.F. Feiner, J. Zaanen, Phys. Rev. B 61, 6257 (2000).

[34] M. Daghofer, D. R. Neuber, A. M. Oleś, W. von der Linden, Phys. Status Solidi $B$ 243, 277 (2006).

[35] F. Mack, P. Horsch, Phys. Rev. Lett. 82, 3160 (1999).

[36] L.F. Feiner, A.M. Oleś, Phys. Rev. B 71, 144422 (2005).

[37] D. Neuber, M. Daghofer, A.M. Oleś, W. von der Linden, Phys. Status Solidi C 3, $32(2006)$.

[38] T. Kimura, K. Hatsuda, Y. Ueno, R. Kajimoto, H. Mochizuki, H. Yoshizawa, T. Nagai, Y. Matsui, A. Yamazaki, Y. Tokura, Phys. Rev. B 65, 020407 (2002).

[39] T. Hotta, Phys. Rev. B 67, 104428 (2003).

[40] D.V. Efremov, J. van den Brink, D.I. Khomskii, Nature Mater. 3, 853 (2004). 